\title{
ON CONTINUITY AND DIFFERENTIABILITY OF THE MAXIMUM VALUES OF FUNCTIONS
}

\author{
V.A. KLYACHIN
}

\begin{abstract}
In this paper we consider functions, which are the maximal values of continuous functions on the families of compact subsets. Such functions are used, for example, in studying the geometric structure of various equilibrium surfaces: minimal surfaces, surfaces of a constant mean curvature, and so forth. Usually, such functions are constructed as the geometric characteristics of the surfaces under study, for instance, as the distance from a point of the surface to a fixed line, as the radius of the circumscribed sphere. One of the key points of this approach is the justification of their continuity and differentiability. This allows us to derive differential relations for the considered functions. In the present paper, the questions of continuity and differentiability are considered in a more general formulation, for topological and metric spaces. In particular, we find the conditions for the mapping of topological spaces $F: X \rightarrow T$ ensuring that a function of the form $\rho(t)=$ $\max _{x \in F^{-1}(t)} g(x)$ is continuous. In addition, for such functions we obtain the conditions guaranteeing that they are Lipschitz and $\delta$-convex in $\mathbb{R}^{m}$.
\end{abstract}

Keywords: metric space, Lipschitz functions, continuity, differentiability, $\delta$-convexity.

Mathematics Subjects Classifications: 26B05, 26B35

\section{INTRODUCTION}

One of the methods for studying minimal surfaces and the surfaces with a prescribed mean curvature is based on using of so-called girth function $\rho(t)$, which is the maximal distance from a point at some straight line to the points of the surfaces lying in the plane orthogonal to this line. For the girth function, various differential inequalities are obtained and integrating them, a certain information on the structure of the surfaces is found out. Such approach was used in works by V.M. Miklyukov, A.D. Vedenyapin, M.V. Privalov, N.V. Loseva, V.G. Tkachev, V.A. Klyachin [1]-[7].

It was shown in work [1] that for the minimal hypersurfaces of tubular form this function is convex down and satisfies the differential inequality

$$
\rho^{\prime \prime}(t) \rho(t) \geqslant(n-1)\left(1+\rho^{\prime 2}(t)\right)
$$

almost everywhere. In works [2]-[7, similar inequalities were obtained for the minimal surfaces of arbitrary codimension, for $p$-minimal hypersurfaces, for the surfaces of a prescribed mean curvature. In all these cases the proof is based on the comparison principle for elliptic operators of mean curvature kind, and the almost everywhere existence of the second derivatives is obtained by using the maximum principle for specially constructed subharmonic functions in the metric of the corresponding surface. We should also mention the classical Hadamard theorem on three circles. This theorem states that for a holomorphic function $f(z)$ defined in the annuli $r_{1} \leqslant|z| \leqslant r_{3}$, for all $r_{1}<r_{2}<r_{3}$, the inequality

$$
\log \left(\frac{r_{3}}{r_{1}}\right) \log M\left(r_{2}\right) \leqslant \log \left(\frac{r_{3}}{r_{2}}\right) \log M\left(r_{1}\right)+\log \left(\frac{r_{2}}{r_{1}}\right) \log M\left(r_{3}\right)
$$

V.A. KLyachin, On CONTINUity and Differentiability of the maXimum Values of FunCtions.

(C)KLYACHIN V.A. 2017.

Submitted May 17, 2016. 
holds true, where $M(r)=\max _{|z|=r}|f(z)|$. This inequality is equivalent to the fact that the function $\varphi(t)=\log M\left(e^{t}\right)$ is convex down for $t \in\left(\log r_{1}, \log r_{3}\right)$. Some generalization of this theorem can be found in [8]. The proof of Hadamard inequality is also based on the maximum principle.

In the present paper we provide the proof of continuity, differentiability and $\delta$-convexity for the functions representing the maximal values of continuous and smooth functions without using the maximum principle or its analogues.

\section{Continuity}

Let $X$ and $T$ be topological spaces and $F: X \rightarrow T$ be some continuous mapping such that for each pre-compact set $K \subset T$ the pre-image $F^{-1}(K)$ is pre-compact in $X$. In particular, the sets $\Sigma(t)=F^{-1}(t), t \in T$, are compact. Let $g: X \rightarrow \mathbb{R}$ be a continuous function, for which we define

$$
\rho(t)=\max _{x \in \Sigma(t)} g(x) .
$$

Theorem 1. Assume that each open covering of the compact set $\Sigma(t)$ is an open covering of $\Sigma\left(t^{\prime}\right)$ for all $t^{\prime}$ in some neighbourhood of the point $t$. If the mapping $F$ is open, then the function $\rho(t)$ is continuous.

Proof. Let $t_{0} \in T$ be a point and $x_{0} \in \Sigma\left(t_{0}\right)$ is such that $g\left(x_{0}\right)=\rho\left(t_{0}\right)$. Let $\varepsilon>0$ be arbitrary. Since the function $g$ is continuous, then for each point $x \in \Sigma\left(t_{0}\right)$ we can define a neighbourhood $U(x)$ such that

$$
|g(x)-g(y)|<\varepsilon \quad \text { for all } y \in U(x)
$$

The set of all such neighbourhoods is an open covering of the compact set $\Sigma\left(t_{0}\right)$. We choose a finite subcovering $U\left(x_{k}\right), k=1, \ldots, m$, in this covering. Then, since the mapping $F$ is open, the set $\cup_{k=1}^{m} F\left(U\left(x_{k}\right)\right)=V\left(t_{0}\right)$ is open. Let $V^{\prime}\left(t_{0}\right)$ be a neighbourhood of the point $t_{0}$, for which the union $\cup_{k=1}^{m} U\left(x_{k}\right)$ is the covering of $\Sigma\left(t^{\prime}\right)$ for each $t^{\prime} \in V^{\prime}\left(t_{0}\right)$. It is clear that for $t^{\prime} \in V^{\prime}\left(t_{0}\right)$ the intersection $\Sigma\left(t^{\prime}\right) \cap U\left(x_{k}\right)$ is non-empty for some $k$. we consider $x^{\prime} \in \Sigma\left(t^{\prime}\right), g\left(x^{\prime}\right)=\rho\left(t^{\prime}\right)$ and $x^{\prime} \in U\left(x_{k}\right)$. Then

$$
\rho\left(t_{0}\right)=g\left(x_{0}\right) \geqslant g\left(x_{k}\right)>g\left(x^{\prime}\right)-\varepsilon=\rho\left(t^{\prime}\right)-\varepsilon .
$$

Since $\Sigma\left(t^{\prime}\right) \cap U\left(x_{k}\right) \neq \emptyset$, there exists $y^{\prime} \in \Sigma\left(t^{\prime}\right) \cap U\left(x_{k}\right)$. Then

$$
\rho\left(t^{\prime}\right)=g\left(x^{\prime}\right) \geqslant g\left(y^{\prime}\right)>g\left(x_{0}\right)-\varepsilon=\rho\left(t_{0}\right)-\varepsilon .
$$

So, for $t^{\prime} \in V^{\prime}\left(t_{0}\right)$ we have

$$
\left|\rho\left(t^{\prime}\right)-\rho\left(t_{0}\right)\right|<\varepsilon \text {. }
$$

Therefore, the function $\rho(t)$ is continuous. The proof is complete.

We consider an example. In the circle $B=\left\{x \in \mathbb{R}^{2},|x|<1\right\}$ we define the function

$$
F(x)=e^{-r} \cos 3 \pi r, \quad r=|x| \text {. }
$$

Then

$$
\Sigma(t)=\{x \in B: F(x)=t\} .
$$

We let $g(x)=x_{1}$. As one can see, the function $\rho(t)=\max _{\Sigma(t)} g(x)$ has a discontinuity at the point

$$
t=e^{-r_{0}} \cos 3 \pi r_{0}, \quad r_{0}=\frac{2}{3}-\frac{1}{3 \pi} \arctan \left(\frac{1}{3 \pi}\right) .
$$

At that we observe that the mapping defined by the function $F(x)$ is not open in the vicinity of the circumference $r=r_{0}$.

Assume now that $X=\left(X, d_{X}\right)$ is a metric space. By $K(X)$ we denote the metric space of compact subsets $K \in X$ equipped with the standard Hausdorf metric

$$
d_{K}\left(K_{1}, K_{2}\right)=\max \left\{\max _{y \in K_{2}} \min _{x \in K_{1}} d_{X}(x, y), \max _{y \in K_{1}} \min _{x \in K_{2}} d_{X}(x, y)\right\} .
$$


Let $F: T \rightarrow K(X)$ be some continuous mapping and $g: X \rightarrow \mathbb{R}$ be a continuous function. We let $\Sigma(t)=F(t), t \in T$.

Theorem 2. If the function $g(x)$ is uniformly continuous, then the function

$$
\rho(t)=\max _{\Sigma(t)} g(x)
$$

is continuous.

Proof. Given $\varepsilon>0$, by the uniform continuity of the function $g(x)$ there exists $\delta>0$ such that if $d_{X}\left(x^{\prime}, x^{\prime \prime}\right)<\delta$, then $\left|g\left(x^{\prime}\right)-g\left(x^{\prime \prime}\right)\right|<\varepsilon$. Let $t_{0} \in T$. Since the mapping $F$ is continuous at the point $t_{0}$, then for $\delta>0$ there exists a neighbourhood $V\left(t_{0}\right)$ of the point $t_{0}$ such that

$$
d_{K}\left(\Sigma(t), \Sigma\left(t_{0}\right)\right)<\delta
$$

holds for each $t \in V\left(t_{0}\right)$.

By the compactness of the sets $\Sigma\left(t_{0}\right)$ and $\Sigma(t)$ there exist points $x_{0} \in \Sigma\left(t_{0}\right)$ and $x^{\prime} \in \Sigma(t)$ such that

$$
g\left(x_{0}\right)=\rho\left(t_{0}\right), \quad g\left(x^{\prime}\right)=\rho(t) .
$$

There exist also the points $y_{0} \in \Sigma\left(t_{0}\right)$ and $y^{\prime} \in \Sigma(t)$ such that

$$
\begin{aligned}
& d_{X}\left(x_{0}, y^{\prime}\right)=\min _{y \in \Sigma(t)} d_{X}\left(x_{0}, y\right), \\
& d_{X}\left(x^{\prime}, y_{0}\right)=\min _{y \in \Sigma\left(t_{0}\right)} d_{X}\left(x^{\prime}, y\right) .
\end{aligned}
$$

By the definition of the Hausdorff distance we obtain

$$
d_{X}\left(x_{0}, y^{\prime}\right)<\delta, \quad d_{X}\left(x^{\prime}, y_{0}\right)<\delta .
$$

Then by inequalities (1) we get

$$
\begin{aligned}
& \rho(t)-\rho\left(t_{0}\right)=g\left(x^{\prime}\right)-g\left(x_{0}\right) \leqslant g\left(x^{\prime}\right)-g\left(y_{0}\right)<\varepsilon, \\
& \rho(t)-\rho\left(t_{0}\right)=g\left(x^{\prime}\right)-g\left(x_{0}\right) \geqslant g\left(y^{\prime}\right)-g\left(x_{0}\right)>-\varepsilon .
\end{aligned}
$$

Thus, for each $t \in V\left(t_{0}\right)$ we have $\left|\rho(t)-\rho\left(t_{0}\right)\right|<\varepsilon$. The proof is complete.

\section{LIPSCHITZ PROPERTY AND $\delta$-CONVEXITY}

Assume that the space $T$ is metric with the metric $d_{T}$.

Theorem 3. Assume that mapping $F: T \rightarrow K(X)$ and the function $g: X \rightarrow \mathbb{R}$ satisfy the Lipschitz condition

and

$$
d_{K}\left(F\left(t_{1}\right), F\left(t_{2}\right)\right) \leqslant L_{0} d_{T}\left(t_{1}, t_{2}\right),
$$

Then the function

$$
\left|g\left(x_{1}\right)-g\left(x_{2}\right)\right| \leqslant L_{1} d_{X}\left(x_{1}, x_{2}\right) .
$$

satisfies the Lipschitz condition

$$
\rho(t)=\max _{x \in F(t)} g(x)
$$

$$
\left|\rho\left(t_{1}\right)-\rho\left(t_{2}\right)\right| \leqslant L_{0} L_{1} d_{T}\left(t_{1}, t_{2}\right) .
$$

Proof. We consider some $t_{1}, t_{2} \in T$ and $x_{1} \in \Sigma\left(t_{1}\right), \rho\left(t_{1}\right)=g\left(x_{1}\right), x_{2} \in \Sigma\left(t_{2}\right), \rho\left(t_{2}\right)=g\left(x_{2}\right)$. We choose $y_{1} \in \Sigma\left(t_{1}\right), y_{2} \in \Sigma\left(t_{2}\right)$ such that

$$
\begin{aligned}
& d_{X}\left(x_{1}, y_{2}\right)=\min _{z \in \Sigma\left(t_{2}\right)} d_{X}\left(x_{1}, z\right) \\
& d_{X}\left(x_{2}, y_{1}\right)=\min _{z \in \Sigma\left(t_{1}\right)} d_{X}\left(x_{2}, z\right) .
\end{aligned}
$$

ince the mapping $F$ is Lipschitz, we have

$$
d_{K}\left(\Sigma\left(t_{1}\right), \Sigma\left(t_{2}\right)\right) \leqslant L_{0} d_{T}\left(t_{1}, t_{2}\right) .
$$


Therefore,

$$
d_{X}\left(x_{1}, y_{2}\right) \leqslant L_{0} d_{T}\left(t_{1}, t_{2}\right)
$$

and

Thus, we obtain

$$
d_{X}\left(x_{2}, y_{1}\right) \leqslant L_{0} d_{T}\left(t_{1}, t_{2}\right)
$$

$$
\rho\left(t_{1}\right)=g\left(x_{1}\right) \geqslant g\left(y_{1}\right) \geqslant g\left(x_{2}\right)-L_{1} d_{X}\left(y_{1}, x_{2}\right) \geqslant \rho\left(t_{2}\right)-L_{0} L_{1} d_{T}\left(t_{1}, t_{2}\right),
$$

and

$$
\rho\left(t_{2}\right)=g\left(x_{2}\right) \geqslant g\left(y_{2}\right) \geqslant g\left(x_{1}\right)-L_{1} d_{X}\left(y_{2}, x_{1}\right) \geqslant \rho\left(t_{1}\right)-L_{0} L_{1} d_{T}\left(t_{2}, t_{1}\right) .
$$

Therefore,

$$
\left|\rho\left(t_{1}\right)-\rho\left(t_{2}\right)\right| \leqslant L_{0} L_{1} d\left(t_{1}, t_{2}\right)
$$

The proof is complete.

Let $\Omega \subset \mathbb{R}^{m}$ be a domain in $\mathbb{R}^{m}$ and $F: \Omega \rightarrow \mathbb{R}^{p}, p<m$, is a $C^{1}$-smooth mapping with $\operatorname{rank} d F=p$. Assume that for each $t \in \mathbb{R}^{p}$ the set $F^{-1}(t)=\Sigma(t)$ is compact in $\Omega$. We consider a $C^{2}$-smooth function $g: \Omega \rightarrow \mathbb{R}$ and we let

$$
\rho(t)=\max _{x \in \Sigma(t)} g(x) .
$$

Theorem 4. The function $\rho(F(x))$ is locally $\delta$-convex, that is, for each point $x_{0} \in \mathbb{R}^{m}$ there exists a neighbourhood $U\left(x_{0}\right)$ and a convex function $v(x)$ such that the function $\rho(F(x))+v(x)$ is convex in $U\left(x_{0}\right)$.

In the proof of the theorem we shall make us of the following lemma.

Lemma 1. Let $h(x), x \in \mathbb{R}^{m}$, be a continuous function such that for some $\alpha \in \mathbb{R}, \alpha>0$, and each point $x_{0} \in \mathbb{R}^{m}$ there exists a quadratic function of the form

$$
r(x)=-\alpha\left|x-x_{0}\right|^{2}+\left\langle x-x_{0}, \beta\right\rangle+h\left(x_{0}\right)
$$

such that $h(x) \geqslant r(x)$ in some neighbourhood of the point $x_{0}$. Then the function $h(x)+\alpha|x|^{2}$ is convex down.

Proof. We have

$$
\begin{aligned}
h(x)+\alpha|x|^{2} & =h(x)+\alpha\left|x-x_{0}\right|^{2}-2 \alpha\left\langle x_{0}, x-x_{0}\right\rangle+\alpha\left|x_{0}\right|^{2} \\
& \geqslant-\alpha\left|x-x_{0}\right|^{2}+\left\langle\beta, x-x_{0}\right\rangle+h\left(x_{0}\right)+\alpha\left|x-x_{0}\right|^{2}-2 \alpha\left\langle x_{0}, x-x_{0}\right\rangle+\alpha\left|x_{0}\right|^{2} \\
& =h\left(x_{0}\right)+\alpha\left|x_{0}\right|^{2}+\left\langle-2 \alpha x_{0}+\beta, x-x_{0}\right\rangle .
\end{aligned}
$$

This inequality shows that the graph of the function $h(x)+\alpha|x|^{2}$ is located above the hyperplane defined by the equation

$$
x_{m+1}=h\left(x_{0}\right)+\alpha\left|x_{0}\right|^{2}+\left\langle-2 \alpha x_{0}+\beta, x-x_{0}\right\rangle,
$$

and in its turn, this means that the function $h(x)$ is convex down.

Proof of Theorem 4. We fix a point $t_{0} \in \mathbb{R}^{p}$. There exists a point $x_{0} \in \Omega$ such that $\rho\left(t_{0}\right)=$ $g\left(x_{0}\right)$. Since rank $d F=p$, the mapping $F$ is open and therefore, the function $\rho(t)$ is continuous by Theorem 1. Let $U\left(x_{0}\right)$ be some neighbourhood of the point $x_{0}$ and $V\left(t_{0}\right)=F\left(U\left(x_{0}\right)\right)$. We consider the function $h(x)=\rho(F(x))$. It is clear that $g(x) \leqslant h(x), x \in U\left(x_{0}\right)$, and $g\left(x_{0}\right)=h\left(x_{0}\right)$. Since the function $g(x)$ is twice continuous, there exists a constant $\alpha>0$ such that for each point $x_{0}$ and an appropriate vector $\beta \in \mathbb{R}^{m}$ we have

$$
h(x) \geqslant g(x) \geqslant-\alpha\left|x-x_{0}\right|^{2}+\left\langle\beta, x-x_{0}\right\rangle+h\left(x_{0}\right)
$$

in some neighbourhood of the point $x_{0}$. According Lemma 1, the function $h(x)+\alpha|x|^{2}$ is convex and hence, $\rho(F(x))$ is $\delta$-convex. 
In the general case, under the assumptions of Theorem 4, it is impossible to prove the differentiability of the function $\rho(F(x))$ at each point. Let us consider an example. In the rectangle $[0, \pi] \times[-\pi, \pi]$ we define the function $g(x)=\cos x_{1} \sin x_{2}$. We let $F(x)=x_{1}$. Then

$$
\rho(t)=\max _{x_{1}=t} g(x)=|\cos t|, \quad t \in[0, \pi] .
$$

Thus, $\rho(F(x))=\left|\cos x_{1}\right|$ and this function is not differentiable at the points of the segment $x_{1}=\pi / 2$.

Theorem 5. Let a $C^{2}$-smooth mapping be such that $F(x)$ the mapping

$$
H(x)=(y, \tilde{x}), y=F(x), \quad \tilde{x}=\left(x_{p+1}, \ldots, x_{m}\right),
$$

is a diffeomorphism of the domain $\Omega$ onto the domain $\Omega^{\prime}=H(\Omega)$. Then the function

$$
\rho(t)=\max _{x \in \Sigma(t)} g(x)
$$

is locally $\delta$-convex.

Proof. Let $x=G(y, \tilde{x})$ be the mapping inverse to $H(x)$. Then the function $h(y, \tilde{x})=g(G(y, \tilde{x}))$ is $C^{2}$-smooth in the domain $\Omega^{\prime}$. At that,

$$
\max _{y=z} h(y, \tilde{x})=\rho(z) .
$$

Then, applying Theorem 4 to the function $h(y, \tilde{x})$ and the mapping $F^{\prime}(y, \tilde{x})=y$, we obtain that the function $\rho(y)$ is locally $\delta$-convex $\mathbb{R}^{m}$. That is, in the neighbourhood $U$ there exists a convex function $v(y, \tilde{x})$ such that the function $\rho(y)+v(y, \tilde{x})$ is convex. Then the function

$$
\max _{(y, \tilde{x}) \in U, y=z}\{\rho(y)+v(y, \tilde{x})\}=\rho(z)+\max _{(y, \tilde{x}) \in U, y=z} v(y, \tilde{x})
$$

is also convex in $\mathbb{R}^{p}$. This completes the proof.

\section{BIBLIOGRAPHY}

1. A.D. Vedenyapin, V.M. Miklyukov. Extrinsic dimensions of tubular minimal hypersurfaces // Matem. Sborn. 131:2, 240-250 (1986). [Math. USSR-Sb. 59:1, 237-245 (1988).]

2. M.V. Privalov. Some properties of the girth function for a tubular hypersurface of a constant mean curvature // in "Abstracts of VI Scientific Conference", Volgograd State Univ., Volgograd, 64 (1989). (in Russian).

3. V.G. Tkachev. On the bore radius for minimal surfaces // Matem. Zametki. 59:6, 909-913 (1996). [Math. Notes. 59:6, 657-660 (1996).]

4. V.G. Tkachev. External geometry of p-minimal surfaces // in "Geometry from Pacific Rim". Walter de Gruyter, Berlin, 363-375 (1997).

5. N.V. Loseva. On some properties of saddle hypersurfaces of tube type // Dokl. Akad. Nauk. 336:4, 444-445 (1994). [Dokl., Math. 49:3, 524-527 (1994).]

6. V.A. Klyachin, V.M. Miklyukov. Maximal tubular hypersurfaces in Minkowski space // Izv. AN SSSR. Ser. Matem. 55:1, 206-217 (1991). [Math. USSR-Izv. 38:1, 203-213 (1992).]

7. V.A. Klyachin. An estimate of length for tubular minimal surfaces of arbitrary codimension // Sibir. Matem. Zhurn. 33:5, 201-205 (1992). [Siber. Math. J. 33:5, 928-931 (1992).]

8. V.M. Miklyukov. Geometric analysis. Volgograd State Univ., Volgograd (2007). (in Russian).

Vladimir Alexandrovich Klyachin,

Volgograd State University,

Universitetsky av. 100,

400062, Volgograd, Russia

E-mail: klchnv@mail.ru 